\title{
Trabajo por proyectos en el urbanismo industrial
}

\section{Aguilar ${ }^{a}$, J. Deltoro ${ }^{a}$, V. Albero ${ }^{b}$ y.M. Montalváa}

a Departamento de Ingeniería de la Construcción y Proyectos de ingeniería civil, Universidad Politécnica de Valencia, (magal001@urb.upv.es, judelso@cts.upv.es,jmonsu@cst.upv.es).

bDepartamento de Mecánica de los medios continuos y teoría de estructuras, Universidad Politécnica de Valencia (valbero@mes.upv.es).

\begin{abstract}
This paper exposes the methodology used in the Urbanism part of the subject "Construction, architecture and industrial urbanism" through the realization of the Urban Planning Project of an economic activity sector in groups of three students combining "project based learning" and "cooperative work".

All the contents taught in the subject are applied in the project in a completely practical way on a real case of an existing industrial urban area and its extension. In addition, working in teams supposes a great resemblance with professional practice,

The work contributes to the acquisition of specific competences of the degree and cross-sectional competences of the Polytechnic University of Valencia. The survey conducted on the opinion of the students about it gives us a positive vision of the obtained results.
\end{abstract}

Keywords: project based learning, cooperative working, urbanism, industrial engineering

\section{Resumen}

Este artículo expone la metodología utilizada en la parte de Urbanismo de la asignatura "Construcción, arquitectura y urbanismo industrial"mediante la realización de un Proyecto de ordenación urbanística de un sector de actividad económica en grupos de tres alumnos que aúna el "trabajo por proyectos" y "trabajo cooperativo".

Se puede destacar que el proyecto consiste en la aplicación de los contenidos impartidos en la asignatura de una manera totalmente práctica sobre un caso real de un polígono industrial y su ampliación. Además, el hecho de realizarlo en equipo también supone una semejanza mayor con la práctica profesional.

El trabajo contribuye a la adquisición de competencias tanto específicas de la titulación como transversales de la Universidad Politécnica de Valencia y la realización de una encuesta sobre la opinión de los alumnos nos aporta una visión positiva de los resultados obtenidos.

Palabras clave: trabajo por proyectos, trabajo cooperativo, urbanismo, ingeniería industrial 


\section{Introducción}

Actualmente, como resultado de la puesta en marcha del Espacio Europeo de Educación Superior (EEES) (Bolonia, 1999), es necesario que los nuevos modelos y técnicas educativas centradas en el alumno se vean incrementadas. Los estudiantes no solo deben adquirir habilidades especificas relacionados con su campo de estudio, sino que además deben también desarrollar otras competencias transversales, altamente demandadas y apreciadas por empresas y empleadores. De hecho, en el Comunicado de Bucharest (2012) quedó explicitada la necesidad de combinar competencias transversales y específicas en el ámbito universitario con el objetivo de contribuir a las demandas actuales del mercado y la sociedad.

El interés en dar a los estudios de ingeniería un enfoque práctico (Luce y Henry, 2002) ha supuesto que, desde el punto de vista universitario, cada día sea más necesario diseñar metodologías que permitan a los alumnos simular ejercicios de la vida profesional. En este sentido la aplicación de innovadoras técnicas de aprendizaje como el aprendizaje basado en proyectos, Project Based Learning PBL (Gwem, 2003), ha permitido la adaptación de la educación superior a este nuevo escenario. El aprendizaje basado en proyectos es una metodología centrada en el estudiante que, en grupos pequeños, se enfrenta al planteamiento y desarrollo de proyectos que pretenden dar solución a una necesidad real. Esta metodología se basa en presentar un contexto lo más realista posible que permita al estudiante simular un escenario de la vida profesional. Concretamente, esta tipología metodológica fue aplicada con éxito en el ámbito de la medicina en los años setenta con el objetivo de conseguir una mayor motivación de los estudiantes (Woods et al., 2000) y se ha aplicado posteriormente a otros ámbitos de ingeniería como la ingeniería civil (Mahendran, 1995), la simulación de elementos finitos (Ural, 2013) o la ingeniería estructural (Tejero et al. 2015). Así, se ha convertido en una metodología docente muy popular durante las últimas décadas que resulta realmente útil para incrementar la motivación de los alumnos.

Hoy en día, la metodología basada en proyectos se ha convertido en una metodología de aprendizaje fundamental en los nuevos modelos de educación superior. De hecho, se han realizado diversos estudios que han revelado sus múltiples beneficios en comparación con las metodologías docentes más tradicionales (Maldonado, 2008). Gracias a esta metodología es posible trabajar en el desarrollo de multitud de habilidades y capacidades transversales como el tratamiento de información de múltiples fuentes, el trabajo en grupo, el pensamiento crítico, la planificación y organización de actividades o la toma de decisiones (de Miguel, 2006). Durante todo el proceso de desarrollo de la actividad los estudiantes tienen que trabajar en grupo, planificar y distribuir tareas, analizar diversas situaciones y definir todas las variables involucradas en el diseño final. Para alcanzar de forma satisfactoria el objetivo marcado, los estudiantes deben adoptar una actitud proactiva e interaccionar con sus compañeros para contrastar ideas y opiniones, llegando finalmente a la toma de decisiones.

Este trabajo presenta una aplicación real del método de aprendizaje basado en proyectos diseñada para los estudiantes de la asignatura Construcción, Arquitectura y Urbanismo Industrial del Máster Universitario en Ingeniería Industrial de la Universidad Politécnica de

(cc) EY-NC-ND 2018, Universitat Politècnica de València

Congreso IN-RED (2018) 
Valencia. En este sentido la actividad desarrollada, utilizando esta metodología, permite una aproximación real de los alumnos al ámbito de los proyectos de urbanismo industrial obteniendo unos excelentes resultados y una buena acogida entre el alumnado.

\section{Contexto}

El proyecto descrito en esta comunicación se desarrolla dentro del Máster Universitario en Ingeniería Industrial en la E.T.S.I. INDUSTRIALES. La asignatura, obligatoria y troncal, en la que se desarrolla es la de Construcción, Arquitectura y Urbanismo Industrial (CAUI, Código: 33806) de 5,25 créditos (Teoría: 2,80. Prácticas: 2,45) constituye la base formativa para alcanzar los objetivos que, en el ámbito de la construcción, la arquitectura y el urbanismo, permiten proyectar, calcular y diseñar polígonos industriales.

El temario de la asignatura permite dividir la misma en dos áreas temáticas diferenciadas, Contrucción y Arquitectura industrial y Urbanismo.

Tabla 1. Unidades didácticas

\begin{tabular}{lll}
\hline $\begin{array}{l}\text { Contrucción } \\
\text { Arquitectura } \\
\text { industrial }\end{array}$ & - & $\begin{array}{l}\text { La arquitectura de la planta industrial: La distribución en planta, } \\
\text { Iluminación natural, Protección contra incendios }\end{array}$ \\
\hline Urbanismo & - & Elementos constructivos: Forjados, Soleras, Cerramientos \\
& - & $\begin{array}{l}\text { Introducción al Urbanismo: El suelo de actividades económicas, } \\
\text { Evolución de los asentamientos industriales, Caracterización del } \\
\text { espacio industrial en la Comunitat Valenciana }\end{array}$ \\
& - & $\begin{array}{l}\text { Legislación y planeamiento en la Comunitat Valenciana } \\
\text { Los modelos - La estructura viaria }\end{array}$ \\
& - & Parcelación - Tipologías de edificación \\
&
\end{tabular}

Ambas partes se desarrollan en paralelo con sesiones tanto teóricas como prácticas. La experiencia que se presenta en esta comunicación se incluye dentro la parte de Urbanismo.

Combinando los contenidos teóricos y prácticos, y con un enfoque docente eminentemente aplicado, se desarrollan un conjunto de temas que permitirán al alumno, de manera autónoma, mediante la realización de cálculos y análisis sobre casos reales, teniendo en cuenta la legislación, las normas y los reglamentos de obligado cumplimiento, abordar el proyecto de análisis y de ordenación urbanística de una instalación industrial.

Esta es una asignatura que integra una buena parte de las capacidades anteriormente adquiridas por el alumno, y que posibilita desarrollar su razonamiento crítico, creatividad e iniciativa, alcanzando a comprender la responsabilidad que tendrá, como profesional, sobre los recursos humanos y materiales puestos a su disposición, individualmente o formando parte de un equipo multidisciplinar, para el completo desarrollo del proyecto de construcción, edificación, infraestructuras y urbanismo, en el ámbito de la ingeniería industrial.

La actividad realizada consiste en el análisis de una zona industrial para su reforma y/o ampliación y el desarrollo de un proyecto de ordenación urbanística de una zona de actividades económicas. 
Las metodologías utilizadas para el desarrollo de la actividad descrita son diversas:

- Aprendizaje basado en problemas. Posee un enfoque educativo orientado al aprendizaje y a la instrucción en el que los alumnos abordan problemas reales en pequeños grupos y bajo la supervisión de un tutor. La agrupación propuesta es de tres alumnos.

- Aprendizaje basado en proyectos: Se plantea en situaciones en las que el alumno debe explorar y trabajar un problema práctico aplicando conocimientos interdisciplinares. En este proyecto se deben aplicar conocimientos previos que los alumnos poseen, así como los nuevos conceptos aprendidos previamente o en paralelo dentro de la asignatura CAUI.

- Trabajo colaborativo o grupal: Se realizan sesiones supervisadas donde los estudiantes trabajan en tareas grupales y reciben asistencia y guía cuando es necesaria.

- Aprendizaje significativo aplicado a un contexto real. La elección de un polígono existente ayuda a comprender los conceptos relacionándolos entre sí y obtener una visión completa.

Las competencias (básicas, generales y específicas) que se trabajan en la parte de Urbanismo de la asignatura e incluidas dentro de la Verificación por el Consejo de Universidades del Máster Universitario en Ingeniería Industria, son las siguientes: CB10(G), CB7(G), CB8(G), ,CB9(G), GE2(G), ,GE8(G), CB6(G), IPC1(E), ,IPC2(E).

Las competencias transversales de la UPV trabajadas en la parte de urbanismo son la siguientes:

Tabla 2. Competencias transversales

\begin{tabular}{|c|c|c|c|}
\hline $\begin{array}{c}\text { Competencias } \\
\text { transversales de la } \\
\text { UPV }\end{array}$ & Actividades & Descripción & $\begin{array}{l}\text { Criterios de } \\
\text { evaluación }\end{array}$ \\
\hline (05) Diseño y proyecto & $\begin{array}{c}\text { Aprendizaje } \\
\text { orientado a } \\
\text { proyectos }\end{array}$ & $\begin{array}{l}\text { Análisis de una zona industrial } \\
\text { para su reforma y/o ampliación } \\
\text { y desarrollo del proyecto de } \\
\text { ordenación urbanística de una } \\
\text { zona de actividades } \\
\text { económicas. }\end{array}$ & $\begin{array}{c}\text { Conjunto de rúbricas } \\
\text { para la evaluación de } \\
\text { las diferentes etapas } \\
\text { del proyecto. }\end{array}$ \\
\hline $\begin{array}{l}\text { (07) Responsabilidad } \\
\text { ética, medioambiental y } \\
\text { profesional }\end{array}$ & $\begin{array}{l}\text { Estudio de } \\
\text { casos. }\end{array}$ & $\begin{array}{l}\text { Análisis de una zona industrial } \\
\text { para su reforma y/o ampliación. }\end{array}$ & $\begin{array}{l}\text { Redacción de } \\
\text { informes. }\end{array}$ \\
\hline $\begin{array}{l}\text { (12) Planificación y } \\
\text { gestión del tiempo }\end{array}$ & Proyectos. & $\begin{array}{l}\text { Adecuada organización y } \\
\text { secuenciación temporal para el } \\
\text { desarrollo del proyecto de } \\
\text { ordenación urbanística de una } \\
\text { zona de actividades } \\
\text { económicas. }\end{array}$ & $\begin{array}{l}\text { Conjunto de rúbricas } \\
\text { para la evaluación de } \\
\text { las diferentes etapas } \\
\text { del proyecto. }\end{array}$ \\
\hline
\end{tabular}

(c) B EY-NC-ND 2018, Universitat Politècnica de València

Congreso IN-RED (2018) 
A parte de los objetivos explícitamente formulados a través de las competencias básicas, específicas y transversales que se han enunciado previamente, la realización del proyecto tambiñen persigue la consecución de otros objetivos no explícitos como el fomentar el trabajo cooperativo, el desarrollo de la comunicación escrita en la redacción de informes y el fomento de un juicio y pensamiento crítico.

\section{Descripción del proyecto}

\subsection{Punto de inicio}

El trabajo de Urbanismo del curso, realizado en equipos de 3 alumnos que se constituyen de forma voluntaria, consiste en el análisis de la situación actual de ordenación urbanística de un sector de actividad económica y en la propuesta de ampliación y de ordenación urbanística, de un sector de actividad económica situado en el mismo municipio, con una extensión delimitada en la información gráfica facilitada. La elección de la localización del mismo es distinta cada curso académico. Para alcanzar el objetivo descrito, se desarrollan las siguientes actividades:

- Estudio de la evolución histórica del urbanismo desde el punto de vista de la configuración de las áreas de actividad económica. CONOCIMIENTO

- Análisis de la realidad del suelo de actividades económicas en el ámbito de la Comunitat Valenciana, como referente inmediato que sirve de modelo al análisis general. CRÍTICA.

- Estudio de las herramientas de planeamiento y de proyecto necesarias para el correcto desarrollo de nuevos sectores de actividad económica, junto con los requerimientos técnicos asociados, para la realización del proyecto básico de implantación. EXPERIENCIA DE PROYECTO

Las sesiones teóricas y prácticas de la asignatura permiten abordar progresivamente el proyecto de ordenación de un área de actividad económica, de acuerdo con el enunciado que se facilita. Mediante la realización de este análisis y propuesta urbanística, se pretende obtener un aprendizaje eficaz, a través de la puesta en práctica de los conocimientos y normativas aprendidas en la parte teórica de la asignatura mediante la reflexión y la experimentación.

\subsection{Análisis}

La primera parte del trabajo consiste en la realización de un análisis de un polígono industrial. Para su realización los profesores y alumnos visitan conjuntamente la zona a estudiar. El departamento organiza una salida desde la Universidad al polígono para permitir a todos los alumnos poder recoger y constatar in situ los datos que posteriormente reflejarán en sus presentaciones. 

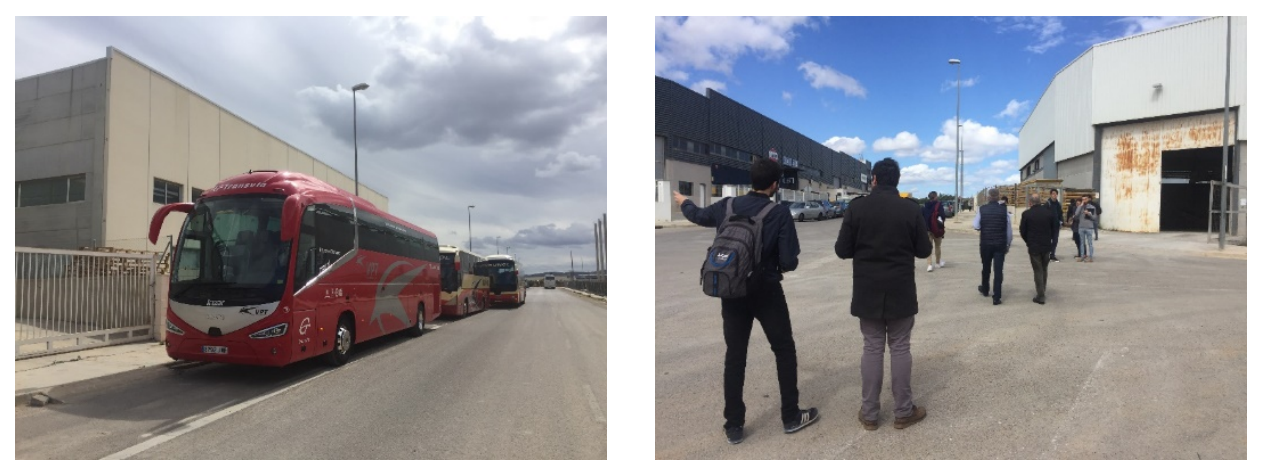

Fig. 1 Visita inicial al área de estudio.

El análisis realizado por los alumnos debe contener la información extraida en unos planos especificados previamente, como la elaboración de un DAFO (debilidades, amenazas, fortalezas y oportunidades, del área objeto de estudio), un reportaje fotográfico y un plano de estructura viaria, usos, parcelación y edificación. Los planos, en formato DIN A3 que los alumnos deben presentar obligatoriamiente son los siguientes:

- A1. Emplazamiento, inserción territorial Y DAFO. E: 1/15.000.

- A2. Composición fotográfica del sector.

- A3. Estructura viaria, usos, parcelación y edificación. E: 1/3.000.

\subsection{Propuesta}

La segunda parte del trabajo consiste en proyectar la ampliación del sector de actividad económica que se ha analizado en la primera parte del curso. Los grupos se mantienen de tres componentes para esta parte del trabajo.

Los alumnos se tienen que adaptar a la superficie prevista para la ampliación establecida por los profesores, de esta forma tratamos de que el ejercicio se asemeje a la realidad. Cuando se va a realizar un proyecto urbano, el promotor, sea público o privado, dispone de un terreno concreto fruto de las expropiaciones o recalificaciones pertinentes.

La propuesta también debe responder a algunos condicionantes que le den verosimilitud: debe adecuarse a la normativa urbanística vigente y se tratará de obtener un rendimiento del suelo razonable. Además, la ordenación debe diseñarse de modo que se produzca una adecuada conexión con el polígono existente y su entorno tanto funcional como formal. Se conectará con las principales infraestructuras viarias y las parcelas y naves proyectadas deben tener unas dimensiones semejantes. La estructura viaria deberá tener una adecuada jerarquización pero no debe comprender más de tres tipos de secciones de calle que deberán diseñar, al margen de posibles vías interiores de servicio. No hay condiciones singulares en relación con la parcelación, ocupación parcelaria, edificabilidad, etc.; se trata de realizar una propuesta libre en el tamaño, número y forma de las parcelas, respetando las reglas formales básicas de composición. Se diseñará la distribución de zonas verdes y aparcamientos y en cuanto a los usos, además del uso industrial, pueden decidir incluir algún equipamiento público o actividades del sector terciario. 
Sin embargo, en cuanto al modo de presentación del proyecto no se permiten tantos grados de libertad. Al igual que en el análisis se establece el número de planos y el contenido que debe haber en cada uno y se aportan las plantillas en formato de autocad.dwg para que trabajen sobre ellas. En la mayoría de los planos se establece la escala a la que se deberá trabajar. Pensamos que los alumnos no están familiarizados con la presentación de proyectos y que darles directrices claras sobre cómo organizar la información les facilita la tarea y pueden centrarse en el proyecto en sí de ampliación de polígono industrial.

Los diferentes planos incluyen tablas en las que los alumnos deberán especificar las superficies globales de cada uso del suelo y porcentajes respecto del total de la zona de ampliación del área industrial. También se cuantificará para cada uso las superficies y porcentajes parciales para completar la definición de los usos del suelo (industrial, terciario y dotacional) el viario y su jerarquización y la caracterización de las zonas verdes. Las tablas permiten comprobar el cumplimiento de los estándares del proyecto

Todos los planos se presentan en tamaño A3. Se establece la presentación obligatoria de los 4 primeros planos, su realización permite alcanzar un 10 de puntuación y se sugiere la realización de dos planos más de carácter voluntario con los que se puede complementar la nota de los anteriores. Los planos obligatorios que se deberán realizar son:

- P1. Propuesta de ordenación general: E: 1/3.000.

- P2. Estructura viaria, usos y espacios verdes: E: 1/3.000.

- P3. Parcelas y ordenanza dibujada: Escala libre.

- P4. Plantas y secciones viarias: E: 1/100, E: 1/200.

Los dos planos optativos son:

- $\quad$ P5. Guías de diseño: E: 1/10, 1/50, 1/100

- $\quad$ P6. Perspectiva general de la propuesta.

\subsection{Rol del profesor / alumno}

Se pretende que el alumno sea capaz de desarrollar autónomamente esta actividad, por lo que el rol del profesor es principalmente de guía, de orientador y supervisor del aprendizaje. Realiza también de transmisor de la información previa necesaria para el desarrollo de la actividad, al inicio de la misma ofreciendo las herramientas y conocimientos necesarios para que la puedan desarrollar posteriormente.

El alumno en este caso debe asumir la responsabilidad de su propio aprendizaje, debe actuar con iniciativa y colaborar y trabajar en equipo con sus compañeros para lograr alcanzar el objetivo y alcanzar las competencias y resultados de aprendizaje esperados.

\subsection{Evaluación}

La asignatura se organiza en dos bloques temáticos: Construcción y arquitectura industrial y Urbanismo. La calificación final de la asignatura se obtiene haciendo media de ambas partes. 
La parte de la asignatura de Urbanismo comprende, a su vez, dos partes. Por un lado se imparten una serie de contenidos teóricos que, además de aplicarse durante la realización del proyecto de urbanismo, se evaluarán mediante un examen escrito individual. La prueba consta de un test de preguntas con respuesta múltiple y de un ejercicio de resolución gráfica y cuenta un $40 \%$ de la nota de Urbanismo. El 60\% de la nota de Urbanismo corresponde al trabajo en equipo. De esta forma se pretende ajustar la calificación para valorar el grado de adquisición de conocimientos real de cada miembro del equipo. De este $60 \%$, la parte de la fase de análisis tiene un peso del $20 \%$ de la nota del trabajo y la fase de propuesta un $80 \%$.

Para la evaluación tanto del análisis como de los proyectos se utilizan rúbricas de corrección que los alumnos pueden consultar durante la realización del trabajo, ya que disponen de ellas desde el principio. La utilización de las rúbricas permite una autoevaluación o evaluación previa que los alumnos pueden realizar a través de unos criterios establecidos y negociados previamente en base a una propuesta realizada por el profesorado al estudiante. Este tipo de evaluación permite incorporar también técnicas de coevaluación que permitan la evaluación significativa del trabajo.

En las rúbricas para la corrección del proyecto se valoran tres aspectos de cada plano y se especifica un porcentaje de peso en la nota de cada uno de ellos. Cada aspecto se puntua eligiendo entre 4 niveles de puntuación: deficiente (0), insuficiente (4), aprobado (7), excelente (10). Se valora con un $10 \%$ de la nota del trabajo a la coherencia de la propuesta, todos los planos de un mismo equipo deberán unificar criterios, gamas de colores, tipos del letra, tamaño de letra.. etc.

\section{Resultados y análisis final}

\subsection{Resultado de los trabajos}

Para los alumnos del Máster en Ingeniería industrial el trabajo propuesto en urbanismo es totalmente nuevo. Los alumnos están habituados a trabajar en proyectos más concretos y técnicos y abordar temas de ciudad es un reto para ellos, tanto por la escala utilizada como por la cantidad de factores que deben tener en cuenta.

Si analizamos las propuestas presentadas por los alumnos se puede constatar que las propuestas alcanzan un nivel de calidad muy satisfactorio. Partiendo de unos mismos criterios y la uniformidad en la forma de presentación se obtienen propuestas muy diferentes entre sí pero válidas y realistas a la hora de afrontar la ampliación del polígono industrial. Para entender mejor los ejemplos aportados correspondientes al curso 2016-17 se muestra un plano de situación del ejercicio planteado ese curso, en Almussafes.

(cc) EY-NC-ND 2018, Universitat Politècnica de València

Congreso IN-RED (2018) 


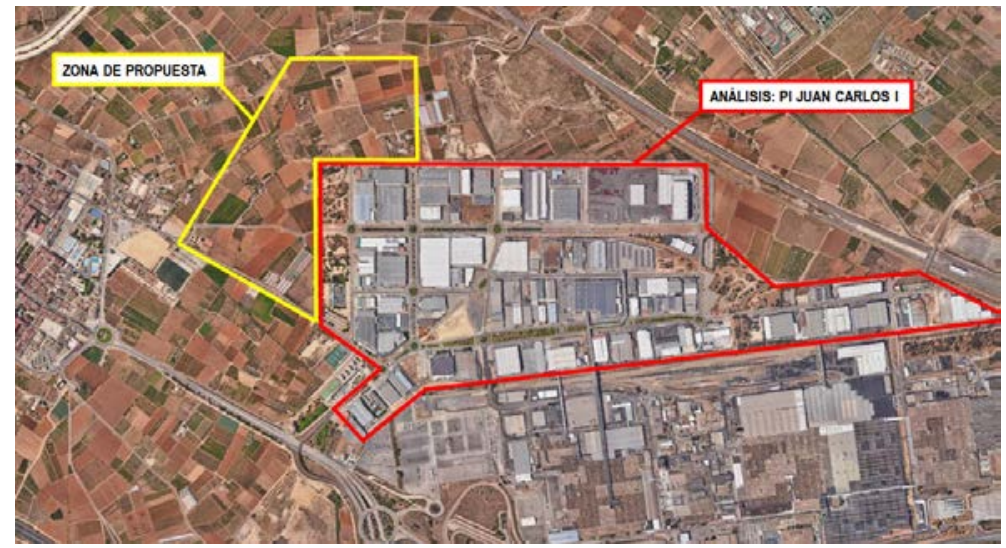

Fig. 2 Plano de situación del proyecto.

Como se puede apreciar a continuación todas las propuestas establecen una clara conexión y continuidad con el polígono existente pero hay diferentes soluciones en cuanto a la situación del viario principal. Mientras algunas de las propuestas lo situaban en la calle más cercana al polígono preexistente otras los llevaban al interior de la zona de ampliación.
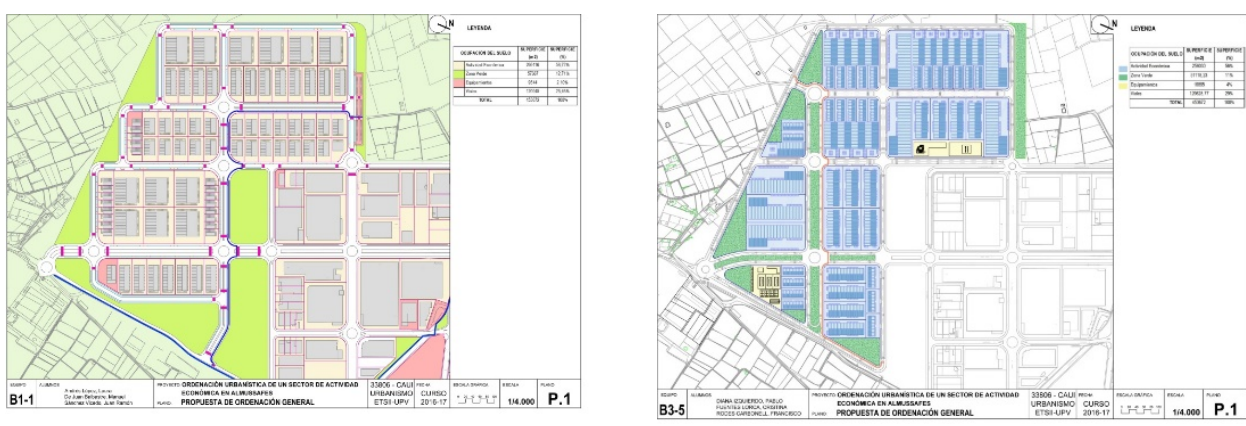

Fig. 3 Ejemplo 1. Plano de ordenación de trabajos de alumnos.

En general, la mayoría de las propuestas continuaban la trama ortogonal del polígono preexistente y utilizaban zonas verdes con formas sensiblemente triangulares para absorber las irregularidades de la parcela. Sin embargo alguno de los equipos optó por girar la trama viaria para ajustarse a la forma de la zona de actuación y resolver las irregularidades en la parte interior de la propuesta, adaptando la forma de las parcelas o introduciendo zonas verdes en estas zonas.
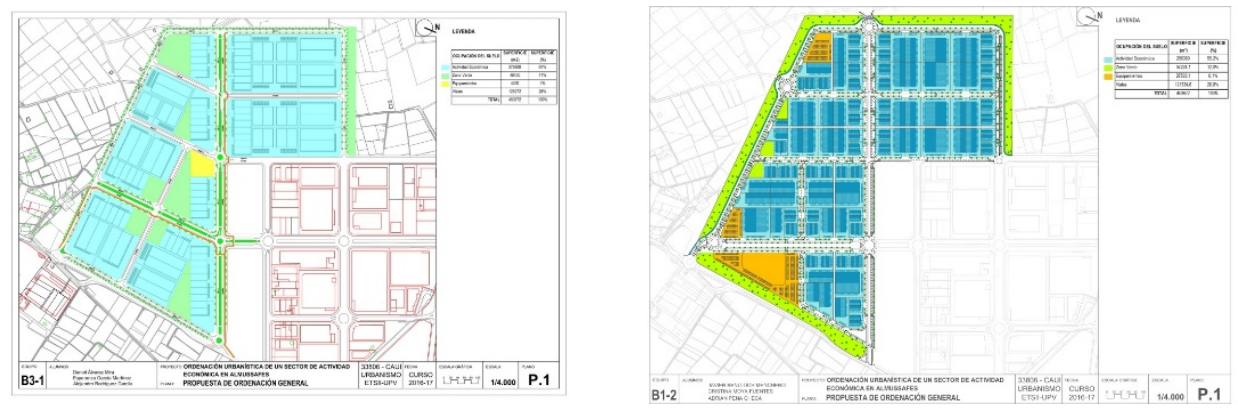

Fig. 4 Ejemplo 2. Plano de ordenación de trabajos de alumnos.

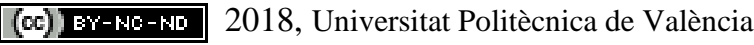

Congreso In-Red (2018) 
En la mayoría de los casos las zonas verdes, caracterizadas como jardín o áreas de juego se han utilizado para completar geometrías irregulares y dejar para las parcelas las formas más ortogonales. Además, la mayoría ha optado por resolver el viario principal mediante boulevar ajardinado. Sin embargo también ha habido equipos que han utilizado las áreas verdes para realizar un anillo perimetral verde y mejorar así su integración con el territorio aportando un colchón verde de transición.

En cuanto a los usos del suelo complementarios al uso industrial las propuestas han sido muy variadas, pero predominan las propuestas de implantación de equipamientos deportivos, guarderías, gasolineras y terciario, principalmente servicios de restauración.

Las propuestas, como ya se ha explicado, incorporaban la definición de las parcelas insutriales y sus ordenanzas especificando todas sus características y representándolo gráficamente.
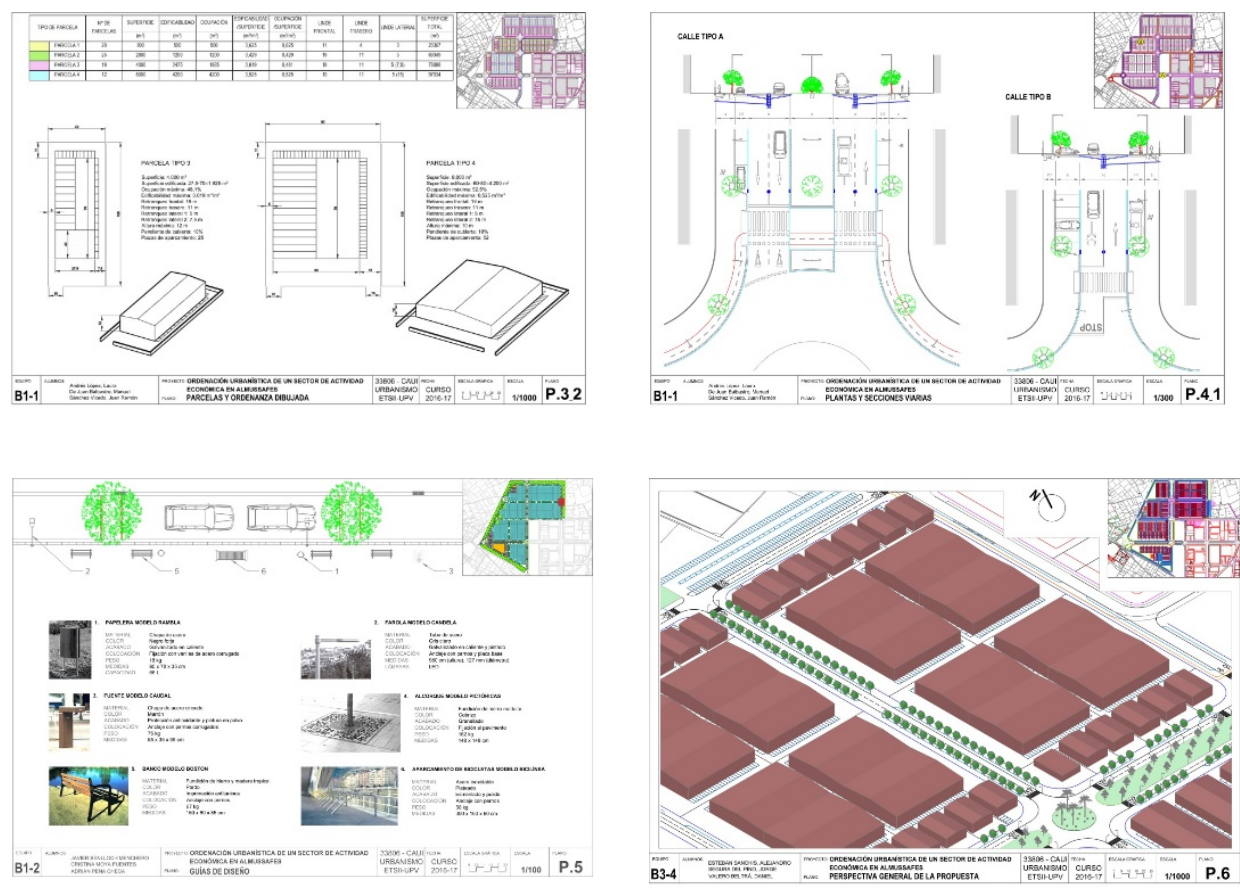

Fig. 5 Ejemplo 3. Planos de parcelación, secciones viarias, guías de diseño y perspectiva de trabajos de los alumnos.

Uno de los aspectos más importantes del trabajo era que se familiarizaran con el diseño de la calle y sus diferentes elementos por lo que se ha insistido en la correcta definición de las secciones viarias de la propuesta.

La mayoría de los equipos optaron por completar el trabajo realizando los dos plamos optativos que se proponía por lo que podemos pensar que los alumnos estaban motivados con el trabajo y se implicaron con el mismo para llegar a un alto grado de definición del proyecto. 


\subsection{Resultado encuestas alumnos}

Finalmente se ha realizado una encuesta de evaluación a los alumnos, con el objetivo de obtener un feedback significativo sobre sus percepciones en la realización del trabajo de la asignatura. Esta encuesta permitirá al profesorado evaluar en nivel de satisfacción de los alumnos con la metodología planteada, así como detectar algunos aspectos a mejorar en los próximos cursos.

La encuesta realizada fue la siguiente:

Q1. ¿Crees que el trabajo ha sido útil para asimilar los conocimientos teóricos en la asignatura?

Q2. El trabajo de la asignatura ¿Te resultó motivador?

Q3. Con las herramientas adquiridas durante la realización del trabajo ¿Crees que serías capaz de realizar un proyecto similar durante tu trayectoria profesional?

Q4. Percepción sobre el grado de adquisición de diversas competencias transversales: Trabajo en equipo y liderazgo, Planificación y gestión del tiempo, etc.

Q5. Nivel de satisfacción general.

La encuesta se realizó de forma voluntaria y totalmente anónima. Para realizarla se empleo la herramienta web GoogleForm que permite una realización asíncrona de la misma y sin necesidad de ninguna infraestructura especial. Las respuestas se recogieron en una escala 15, siendo 5 una valoración totalmente positiva, 3 un nivel medio y 1 una valoración totalmente negativa. Cabe destacar que se recogieron un total de 68 respuestas. En la figura que se muestra más adelante se indican los resultados de algunas de las cuestiones realizadas.

En general puede concluirse que más del $80 \%$ de los alumnos tienen una percepción positiva general sobre la realización del trabajo (niveles 3-4-5) y más del 60\% la perciben de forma muy positiva (niveles 4-5).

Q1

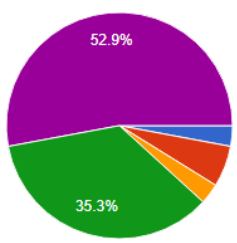

Q3

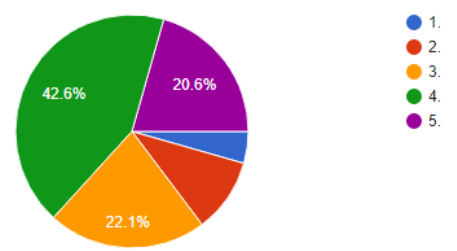




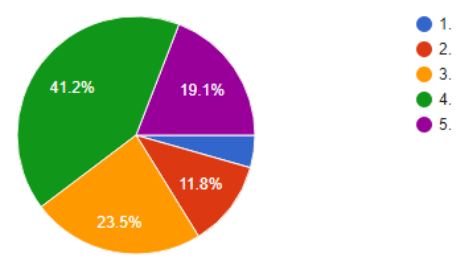

Fig. 6 Resultado de algunas de las cuestiones planteadas a los alumnos.

\section{Conclusiones}

Como ya se ha explicado, el desarrollo de la parte de urbanismo de la asignatura consiste en dos partes relacionadas pero claramente diferenciadas: la parte de teoría que se evalúa mediante una prueba escrita individual y el trabajo en equipo que tiene un mayor peso en la evaluación. Este artículo trata de valorar el resultado de la metodología utilizada en la realización del Proyecto de ordenación urbanística de un sector de actividad económica en equipos de tres alumnos.

Se puede destacar que el trabajo consiste en la aplicación de los contenidos impartidos en la asignatura de una manera totalmente práctica sobre un caso real de un polígono industrial y su ampliación. Además, el hecho de realizarlo por grupos también supone una semejanza mayor con la práctica profesional ya que, en muchos casos, estos proyectos se realizan en equipos multidisplicinares.

Aunque el mencionado trabajo se realiza en la asignatura desde hace varios rompe con la metodología tradicional de enseñanza de clase magistral y incide en un punto de vista aplicado mucho más práctico mediante el "trabajo por proyectos”.

Nuestra experiencia nos hace pensar que el proyecto que realizan los alumnos es capaz de motivarlos y contribuye a que fijen el aprendizaje de los contenidos aprendidos. Los alumnos se implican con el trabajo y se imponen un alto nivel de autoexigencia obteniendo trabajos de considerable calidad. En este sentido la realización del proyecto contribuye a la adquisición de las competencias específicas objetivo de la asignatura.

Además, la metodología utilizada en el trabajo de "trabajo cooperativo" refuerza varias competencias transversales de la Universidad Politécnica de Valencia.

Como ya se ha comentado, las encuestas realizadas nos confirman esa percepción y el grado de satisfacción de los alumnos, tanto en cuanto a la motivación en la realización del trabajo, como en cuanto a lo aprendido, es bastante positivo.

\section{Referencias}

BOLONIA COMUNICADO (1999). EHEA Bolonia Ministerial Conference. [Online] Available:

<http://media.ehea.info/file/Ministerial_conferences/02/8/1999_Bologna_Declaration_Engl ish_553028.pdf> [Consulta: Marzo 2018]

(cc) EY-NC-ND 2018, Universitat Politècnica de València

Congreso IN-RED (2018) 
BUCHAREST COMUNICADO (2012). EHEA Bolonia Ministerial Conference. [Online] Available:

$<$ http://media.ehea.info/file/2012_Bucharest/67/3/Bucharest_Communique_2012_610673.p df> [Consulta: Marzo 2018]

DE MIGUEL, M. (2006). Metodologías de enseñanza y aprendizaje para el desarrollo de competencias. Orientaciones para el profesorado universitario ante el Espacio Europeo de Educación Superior. Alianza Ed.

GWEN, S. (2003). Project-based learning: a primer. Technology and Learning, 23(6), 2030 .

LUCE, L. and HERNY, W.P. (2002). Public awareness of civil engineering and the infrastructure. Journal of Professional Issues in Engineering Education and Practice, 128(4), 165-166.

MAHENDRAN, M. (1995). Project-based civil engineering courses. Journal of Engineering Education, 84(1), 75-79.

MALDONADO, M. (2008). Aprendizaje basado en proyectos colaborativos. Una experiencia en Educación Superior. Revista de Educación, Laurus, 14(28), 158-180.

TEJERO, J., RAMÍREZ, E., MATA, F. y HARAFI, J. (2015). La experiencia del aprendizaje basado en problemas y trabajos proyectuales en las asignaturas de Ingeniería de Estructuras en la Escuela de Ingeniería de Almadén. Innovación educativa en las enseñanzas técnicas. 2, 615-627.

URAL, A. (2013). A hands-on finite element modeling experience in a multidisciplinary project-based freshman course. Computer Applications in Engineering Education, 21(2), 294-299.

Verificación por el Consejo de Universidades del Máster Universitario en Ingeniería Industrial. [Online] Available:

http://www.upv.es/entidades/AEOT/menu_urlc.html?/entidades/AEOT/infoweb/aeot/info/U 0653022.pdf [Consulta: Marzo 2018]

WOODS, D.R., FELDER, R.M., RUGARCIA, A. and STICE, J.E. (2000). The future of engineering education. Developing critical skills. Chemical Engineering Education, 34(2), 108-117. 